(C) 2020 IEEE. This is the author's version of the work. It is posted here for your personal use. Not for redistribution. The definitive Version of Record was published in IEEE Design \& Test, doi: 10.1109/MDAT.2020.3006799.

Personal use of this material is permitted. Permission from IEEE must be obtained for all other uses, in any current or future media, including reprinting / republishing this material for advertising or promotional purposes, creating new collective works, for resale or redistribution to servers or lists, or reuse of any copyrighted component of this work in other works.

DOI: 10.1109/MDAT.2020.3006799. 


\title{
Intelligent Chargers Will Make Mobile Devices Live Longer
}

\author{
Alma Pröbstl, Bashima Islam, Shahriar Nirjon, Naehyuck Chang, Samarjit Chakraborty
}

\begin{abstract}
Battery aging is increasingly becoming a major concern in mobile devices such as laptops or smartphones and often results in premature device replacement. While previous studies have shown that improved charging strategies can increase cycle life, most common chargers do not sufficiently consider battery health. In this perspective paper, we give an overview of recent advances made in battery-health-aware charging and highlight the benefits of making chargers more intelligent to improve the cycle life of different battery-powered devices. In particular, we quantify the potential benefits that intelligent chargers will have and outline possible research directions to make them such.
\end{abstract}

Index Terms-battery managment, mobile devices, smartphones, battery health management, battery charging, intelligent charging

\section{INTRODUCTION}

Battery aging, i.e., the loss of capacity and internal resistance growth, is a big concern in mobile devices such as laptops, tablets or smartphones. Such devices are becoming more feature-rich, thereby having higher power demands and more expensive and bigger batteries. A large fraction of the cost of such devices - and increasingly more so in the future - will be for their batteries. People have also become more reliant on devices like smartphones. As a result, they are subjected to more charge/discharge cycles compared to older phones, which increases battery aging. Battery aging results in premature device replacements and disposal of whole, otherwise functioning, devices. Not only is the resulting electronic waste poisonous for the environment, but also worldwide lithium reserves required for the manufacturing of new batteries are

Alma Pröbstl is a Research Associate at the Technical University of Munich, Chair of Real-Time Computer Systems, Arcisstr. 21, 80290 München, Germany. E-mail: alma.proebstl@tum.de

Bashima Islam is a Graduate Student of Computer Science at the University of North Carolina at Chapel Hill, Department of Computer Science, 201 S. Columbia St., Chapel Hill, NC 27599-3175, United States. Phone: +1 919-338 9160. E-mail: bashima@cs.unc.edu

Shahriar Nirjon is an Assistant Professor of Computer Science at the University of North Carolina at Chapel Hill, Department of Computer Science, 201 S. Columbia St., Chapel Hill, NC 27599-3175, United States. E-mail: nirjon@cs.unc.edu

Naehyuck Chang is a Professor of Electrical Engineering at the Korea Advanced Institute of Science and Engineering, School of Electrical Engineering 291 Daehak Road, Yuseong-Gu, Daejeon 34141, Korea. Phone: +82 (42) 3507434. Fax: +82 (42) 350-7634. Email: naehyuck@ cad4x.kaist.ac.kr

Samarjit Chakraborty is a Professor of Computer Science at the University of North Carolina at Chapel Hill, Department of Computer Science, 201 S. Columbia St., Chapel Hill, NC 27599-3175, United States. Phone: +1 919590-6038. Fax: +1 919-590-6105. E-mail: samarjit@cs.unc.edu

Manuscript received June 26, 2019; revised June 17, 2020. limited. Moreover, the replacement of a device where the processor speed is not the limiting factor, but the insufficient run-time of the battery is, is economically undesirable from the consumer's perspective. In this perspective paper, we discuss how making chargers more intelligent can alleviate this battery aging problem and what challenges lie along this path.

\section{A. Battery replacement}

Until recently, the replacement of mobile devices was largely triggered by insufficient processor speed. But now battery capacities w.r.t. a processor's needs have decreased to a level that it becomes a reason for device replacement. As smartphone technologies slowly tend to plateau off, this trend will become much more prominent. Recently some manufacturers had to slow down smartphones to account for aging batteries.

Non-removable batteries allow a thinner device design. However, non-removable batteries discourage users from replacing batteries. Instead, the users opt for replacing the whole device. This observation is supported by a recent survey [1], which shows that very few users have ever replaced their smartphone (8\%) or laptop (13\%) batteries. Instead, otherwise perfectly working devices were discarded in drawers. Recycling such devices is costly and the necessary infrastructure is still largely unavailable.

\section{B. Aging factors}

The battery State-of-Health ( $\mathrm{SOH}$ ) or cycle life is commonly quantified as the number of complete charge/discharge cycles the battery can conduct before its end of life is reached, i.e., its capacity decreases to less than $70 \%$ or $80 \%$ of its original capacity. Battery aging results in reduced available capacity and increased resistance growth. Factors contributing to battery aging are high average State-of-Charge (SOC), large SOC swing, too low or high cell temperatures, and the charge/discharge current. It has been shown that intelligentlycontrolled measures such as usage-dependent reduction of charge levels, modulating the charging speed, and charging at appropriate times can effectively mitigate battery aging.

\section{Usage-related aging}

But most users are unaware of how to improve the battery health of their devices. They are often stored with unnecessarily high SOC, which increases aging. Typical scenarios are (i) the use of battery-powered charger cases that keep the internal phone battery at a fully-charged level, (ii) charging 
of phones during the night, (iii) using laptops in their docking station during most of the time, (iv) using wireless chargers that encourages users to leave their phones on charging pads to always have a fully-charged battery.

The survey in [1] revealed that $27 \%$ of users only charge when receiving a low-battery warning. This results in a detrimental high SOC swing. Also, only less than half of all users realize that keeping devices connected to the charger for long durations might damage the battery [1]. Hence, most users lack the necessary background on Li-ion aging and factors influencing it, and instead only maximize their usage comfort. Fortunately, the potential for improved battery-health-aware usage exists. $25 \%$ of smartphone users find charging times of more than $2 \mathrm{~h}$ acceptable [1], hence a lower charging current could be used. $36 \%$ of smartphone users follow a fixed charging routine, which could also be exploited by intelligent chargers. Besides, more than half of the users expect manufacturers to ensure better battery life even if that results in increased device sizes. This raises the question of whether we can automate mechanisms for improving battery health, without users having to consciously adopt good practices?

\section{Intelligent chargers}

Intelligent chargers are an answer to this question and can slow-down battery aging, without compromising userexperience, i.e., the battery should have sufficient charge whenever needed, but not too much more to accelerate aging. Similarly, while bad w.r.t. aging, since many users might appreciate faster-charging with a higher current, an intelligent charger can marry user convenience with aging prevention by dynamically selecting the charging current based on predicted future usage. How such a prediction could be done lies at the crux of the charger's intelligence. As an example, it could be based on the device's usage pattern and location data.

\section{E. Objective and outline}

Several prior studies have dwelt on battery-health-aware charging [2], [3], [4], [5], [6]. However, commercial solutions are not yet mature. In what follows, we give an overview of the advances made on the problem of overnight charging and its effects on aging, e.g., the detrimental long periods of fullcharge levels. By delaying the charging until shortly before the device is to be unplugged, the average charge level can be lowered and aging can be mitigated. In the future, we foresee several developments in the domain of intelligent chargers. In this paper, we hypothesize what some of these could be, and point out possible research directions. Building on our previous work, we discuss the design of intelligent chargers that delay charging and select the target SOC, for different types of battery-powered devices. The intelligence broadly relies on predicting both, the future usage of the device and also the availability of charging options during the day. We conclude by discussing different open issues.

\section{EXISTING SOLUTIONS}

Manufacturers seem to prefer selling new devices over battery aging mitigation. However, competition for customers will

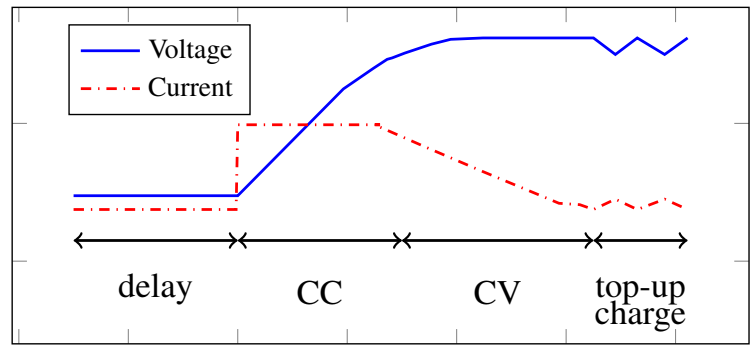

Time

Fig. 1. Conventional CC-CV charging protocol.

increase the importance of battery life. Available battery-aging mitigation approaches range from monitoring to fully codesigned hardware and software solutions. However, existing solutions often impact the user-experience or only target single control knobs.

\section{A. Conventional charge protocols}

Present chargers follow the so-called CC-CV protocol, see Figure 1. First, the device is charged with constant current (CC) to a certain threshold voltage, and then the voltage is kept constant during the constant voltage $(\mathrm{CV})$ phase, where the current is slowly reduced until full-charge is reached. If the device continues to remain plugged, then self-discharge is compensated when the SOC falls below a specified threshold.

\section{B. Battery-health-aware charging}

Control knobs presented in related literature that mitigate battery aging during the charging process are (i) delayed charging [2] also in combination with (ii) reduced charging current [4], [5] and (iii) voltage relaxation periods [6]. So far a joint optimization of delay length, charge current, charge duration and voltage relaxation phase length has not yet been proposed. Intelligence or prediction techniques are required to determine these lengths and durations.

Cycle life gains reported are up to $46.2 \%$ [4] with optimal charging current and delay, and $36 \%$ on an average for adjusted voltage relaxation [6]. If the charge delay is combined with adjusted SOC then the cycle life is almost doubled [2]. These significant gains show the potential of intelligent chargers.

\section{Smartphone apps}

Most existing apps that target battery aging mitigation only insufficiently or inconveniently address battery health. Browsing the app store, we find apps that (i) monitor the remaining charge capacity, (ii) display the cycle count, or (iii) alarm the user to unplug the phone to avoid high SOC levels. Such apps lack intelligence and therefore, decrease the quality of userexperience while not even achieving optimal battery aging mitigation. One reason for this lack of satisfactory solutions is that the access to the charger chip is restricted in common operating systems. Furthermore, some apps require root access thereby making the installation difficult for the common user. 


\section{Dedicated manufacturer solutions for smartphones}

Recently, few manufacturers - e.g., Apple and Sony from 2019 and 2017 respectively - started including intelligent battery cycle-life-enhancing features into their devices.

Learning from past charging routines, the last $10 \%-20 \%$ of charging is delayed to until just before the user unplugs the phone.

In case the user deviates from past routines, the device will be left with around $80 \%$ of its full SOC. Yet, this strategy does not exploit the full potential of intelligent charging. In particular, prediction-based adjustment of the charging current, and the charge limit - i.e., whether only the last $20 \%$ or more should be delayed - is still missing.

\section{E. Joint hardware and software}

A major difficulty when implementing intelligent battery charging solutions stems from the need for real-time diagnostics and data collection. Electrochemical Impedance Spectroscopy (EIS) is one way of obtaining battery parameters to determine battery health. Co-designed hardware and software solutions for real-time measurement are rare. The company Qnovo offers such a solution, but its algorithms are closed source. However, we conclude from their webpages and patents that intelligent algorithms are used to determine charging currents and relaxation times from user behavior.

\section{F. Learning from electric vehicles}

In a broader sense, Electric Vehicles (EVs) are mobile devices too. Battery-degradation-aware intelligent charge management in EVs could rely on information and prediction techniques similar to those used in other devices like smartphones. Hoke et al. [7] report that slow charging and delayed charging significantly extend the cycle life of EV batteries. In particular, the unplug time - if it can be predicted - can be used for intelligent charging. In addition to predictions on usage, intelligent systems management, such as when to switch on/off the air conditioner in the vehicle, is also very helpful.

In particular, Vatanparvar et al. [8] found that intelligent control of the heating, ventilation, and air conditioning systems can improve the battery cycle life by $14 \%$ on average.

\section{G. Charging a hybrid energy storage}

In addition to charging based on usage prediction, Hybrid Energy Storage Systems (HESSs) consisting of, e.g., a Liion battery and a supercapacitor have also been proven to effectively mitigate battery aging. HESSs require an intelligent charge management strategy to distribute the charge and discharge current between the battery and the supercapacitor. In such strategies, the supercapacitor effectively serves as a buffer to reduce the average SOC and the SOC swing of the battery. Here, one possible strategy [9] is to satisfy the stable part of power supply and demand by the Li-ion battery while the supercapacitor deals with the spiky part with high variations. With this strategy not only cycle life is improved but also cycle efficiency, i.e., the deliverable amount of battery charge in a single cycle is increased. Charge migration between battery

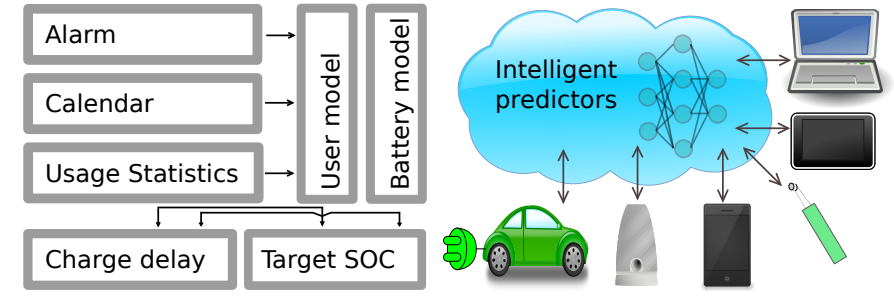

Fig. 2. Intelligent charging prolongs the cycle life of intelligent mobile devices.

and supercapacitor in periods of low power demand facilitates the reduction of the average SOC of the battery. In order to optimally charge or discharge the supercapacitor to minimize high discharge currents from the battery, prediction of the load power is again necessary. With such a combination, gains of up to $4.82 \times$ longer cycle life, compared to battery-only setups, were achieved [9].

As an additional benefit, such HESSs help mitigate aging when using under-dimensioned chargers, that may be lightweight and portable, but do not fulfill peak power demands [3]. Intelligent chargers augmented with such HESSs, therefore, meet the portability requirements, while also addressing battery aging concerns.

\section{The FUtURE OF INTELLIGENT CHARGERS}

Current battery-health-aware charging approaches have only partially leveraged the possibilities to prolong cycle life. In the following, we discuss possible future developments in the domain of intelligent chargers. Towards this, we examine a novel intelligent charging solution, in Figure 2, which (i) makes use of multiple data sources, (ii) connects several intelligent devices, and (iii) combines control knobs for battery health.

\section{A. Intelligent charging of smartphones}

We envision intelligent chargers that monitor a user's smartphone usage and fine-tunes its charging schedule. How would such monitoring work? Monitoring smartphone usage has been investigated in other contexts. Footprint tools have been proposed to capture usage context in Android smartphones [10], where usage context includes battery level, battery health, charging status, screen status, network activities, memory, and CPU usage. Usage context has been used to identify the charging habits of users to identify opportunistic data upload time slots, to evaluate the impact of charging cycles on battery life, and to develop mechanisms to avoid overcharging [11]. Moreover, many current smartphones provide the user with hourly usage and charging information, as shown in Figure 3 a). As many users charge their phones overnight, alarm settings and such usage statistics can be exploited for delayed charging and for determining target SOC [2]. Such measures could significantly reduce the time a battery remains in a highcharge state, thereby help mitigate battery aging.

Together with the usage context, intelligent chargers will also have to account for the availability of charging opportunities. Along with charging behavior, the location of the user 
plays a crucial role here and needs to be predicted to determine whether a charger will be available when needed. For example, one will not be able to charge the phone while going on a run in the park. Failing to incorporate such information will lead to inconvenience, resulting in the user abandoning any aging mitigation efforts in favor of convenience. By combining location data with calendar events, a good prediction on the availability of charging facilities is possible.

\section{B. Intelligent charging of multiple devices}

Smartphone usage and location data can be combined with usage statistics of other consumer electronics, e.g., laptop, tablet, electric toothbrush, smart speaker, and EV, to determine their respective use times and optimize the charging of the battery of each device. Leveraging the correlations between device usage profiles, and the location of the devices, the required charge levels for each device at any specific time of the day can be estimated. By combining activities across multiple electronic devices - a good example being the smartphone, laptop and EV - it will be possible to develop a personalized profile for a user, which can then be exploited to determine the optimal charging profiles for each of these devices.

By taking into account joint device statistics, we will increase the confidence in the predictions on usage and the availability of charging options. Examples of this could be: (i) During a meeting entered in the calendar, the user's EV is likely to remain unused while the laptop requires a sufficiently charged battery. (ii) While the toothbrush is in use in the evening, the user's EV is likely to remain unused for a longer period. (iii) The EV is likely to be used shortly after the toothbrush was used in the morning.

Finally, reinforcement learning is a promising technique for learning both - the usage patterns of a device, and its available charging options. From these, the charger can intelligently plan a charging pattern that includes both charging times, and also currents, while maintaining sufficient backup to account for unlikely events. The goal of the charging pattern would be to reduce aging by (i) charge delay, (ii) lower target charge SOC, and (iii) adjusted charge current. Additionally, implementing a HESSs and the associated intelligent control can further prolong cycle life, while retaining the necessary conveniences.

\section{Results for optimized charging of multiple devices}

Figure 3(c) shows the cycle life gains for smartphones from introducing charge delays and lower charging SOC [2]. The bars represent the average simulated cycle life resulting from looping two-week usage recordings of nine different users. The usage recordings are looped until $80 \%$ of the initial battery capacity remains. The error bars show the distance between maximum and minimum cycle life achieved for the most and the least healthy battery usage. Here, the blue bar shows the original profiles, and the red bars show the cycle life that results from maximum delay, lowest possible target SOC, and the combined measures. The green bars use a moving average predictor from [2]. Notably, all measures significantly improve cycle life, and the predictors are close to ideal performance.
In the future, the combined operation of such individual predictors will allow intelligent charging of multiple devices by exploiting their correlated usage statistics as described above. In Figure 4, we present potential cycle life gains of a multidevice optimized charging regime assuming perfect prediction. We assume generic usage profiles for a user who owns a smartphone, business laptop, and uses an EV. The devices adapt their charging behavior based on a joint database.

Let us assume the following scenario. Under normal usage, the smartphone is charged overnight and unplugged at $6 \mathrm{am}$. The user drives the EV to work from 7-8 am. The EV remains plugged throughout the day at the charging facilities provided by the employer. The first meeting starts at $8 \mathrm{am}$, and the laptop that is usually kept in a docking station is unplugged and discharged. Afterward, the laptop is returned to the docking station and charges again. A second meeting takes place from 2-4 pm, and the laptop discharges. At $5 \mathrm{pm}$, the user drives home using the $\mathrm{EV}$, which then charges overnight.

We maintain a $25 \%$ lower reserve SOC and determine the upper charging limits and charge delays from usage statistics, alarm and calendar entries. Of course, many dependencies exist: E.g., if the laptop remains at the work desk, it starts charging when the EV leaves home. Otherwise, it is charged after the phone is unplugged. The EV starts charging in the morning, once the phone is unplugged and for the after-work trip, once the laptop is back on the docking station. Note that we assume fast charging for the EV both at residential as well as work parking. However, the scenario could easily be changed to slower charging, e.g., using the phone unplug time minus the expected charging duration.

We simulate battery aging (i) in the original scenario and (ii) assuming perfect predictors. In particular, devices without intelligent charging would remain at detrimentally high SOC most of the time. They can achieve significant gains of up to 7 times longer cycle life as shown in Figure 4 . The smartphone that has a higher usage can achieve a significant gain of more than doubling its cycle life compared to conventional charging.

This simulation shows potential gains and encourages further research on intelligent inter-device charging. We have performed this simulation based on artificial usage profiles and assumed perfect knowledge of unplug times and the required charge. In the future, we plan to perform user studies to get a better understanding of the typical charging behavior of different device classes and will evaluate the potential for improvement for classes of users.

\section{OPEn Challenges}

In this section, we highlight the main open challenges of designing an intelligent charger.

\section{A. Information extraction}

Designing an intelligent charger requires some necessary information for decision making. This information includes the unplug time, the target SOC, SOH, usage context and location. Most phone manufacturers and operating system designers do not provide a sufficiently accessible and standardized interface to delay charge, change the charge current, or the target SOC. 


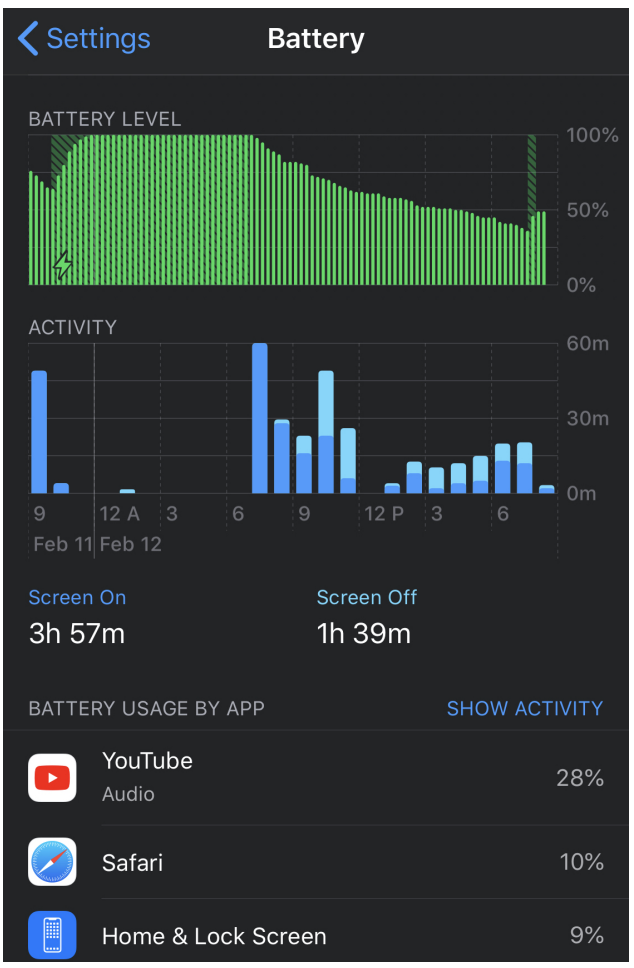

(a) iPhone usage and charging information

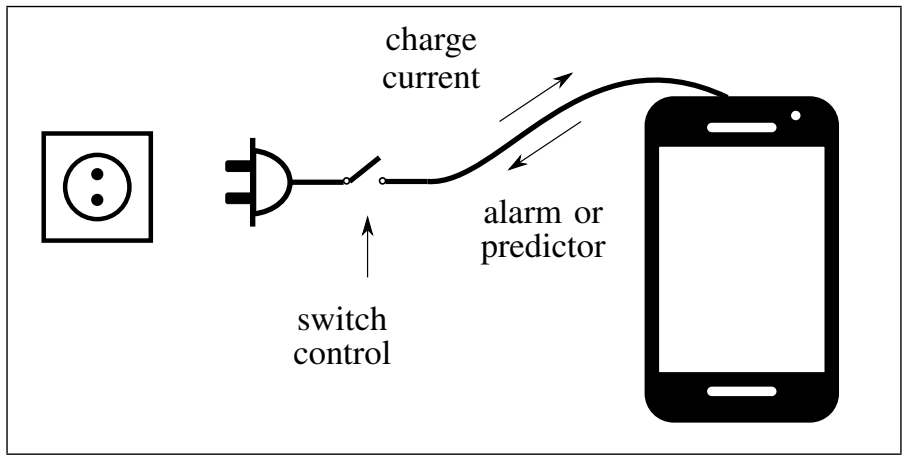

(b) Remote controlled switch for intelligent charging control [2]

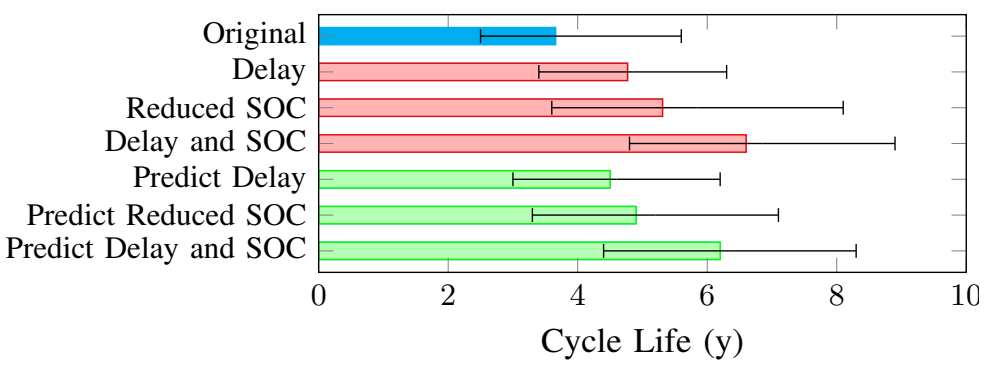

(c) Gains of intelligent smartphone charging achieved by [2]

Fig. 3. Intelligent charging significantly improves the battery cycle life of smartphones.

As the systems do not readily provide such information, either predictors can estimate these values, or the user can provide such information when plugging-in the phone, laptop, tablet or EV. Considering the convenience of the user, the former is more feasible and thus an accurate predictor will make a big difference.

For example, lightweight and low-cost $\mathrm{SOH}$ estimation solutions will allow further optimization of the aging mitigating strategies by providing online feedback of available effectiveness. To estimate $\mathrm{SOH}$, Doan et al. [12] introduce an intelligent charger that features an online impedance spectroscopy technique. However, the charger does not include any additional intelligence as discussed above. Furthermore, the charger requires additional hardware which results in higher cost. Hence, collecting SOH of batteries without additional hardware is crucial.

Moreover, the computation and energy cost of obtaining this information necessitates it to being minimal to avoid additional battery drain.

\section{B. User comfort and acceptance}

The major challenge of designing an intelligent charger is the comfort and convenience of the user. A user might opt for more conservative choices due to the lack of understanding of battery aging factors. Thus, any intelligent charger has to be user-friendly and cannot compromise convenience. To illustrate, most of the users feel more comfortable having a fully charged battery even if they do not foresee using it fully before the next charging opportunity. Therefore it is a challenge to convince a user to not overcharge. To address user satisfaction while not unnecessarily overcharge, a solution could be to display a quantized battery charge level - such as high, medium, or low in the battery icon on the screen - instead of a percentage of full charge. Intelligent chargers could dynamically change the boundaries of these quantized levels based on a specific user's usage and information from other data sources. For example, a user whose battery is never drained below $50 \%$ can have $60 \%$ as high. On the other hand, a user who drains the battery below $10 \%$ daily should see $80 \%$ as a high level.

Moreover, raised user awareness would also increase the pressure on manufacturers, as the demand for sustainable, battery health-friendly devices continues to grow.

\section{Charging protocol}

The charge protocol design needs refinement as the appropriate length of $\mathrm{CC}$ and $\mathrm{CV}$ phases, the amount of charge current, and required voltage relaxation times may depend on the specific battery. However, these details are not exclusively included in the datasheets from battery manufacturers. Though online measurement and related adaption have been proposed for such scenarios, they are expensive due to the additional built-in hardware. Designing an optimized charge protocol and solving the necessary optimization problems is only part of the challenge. 


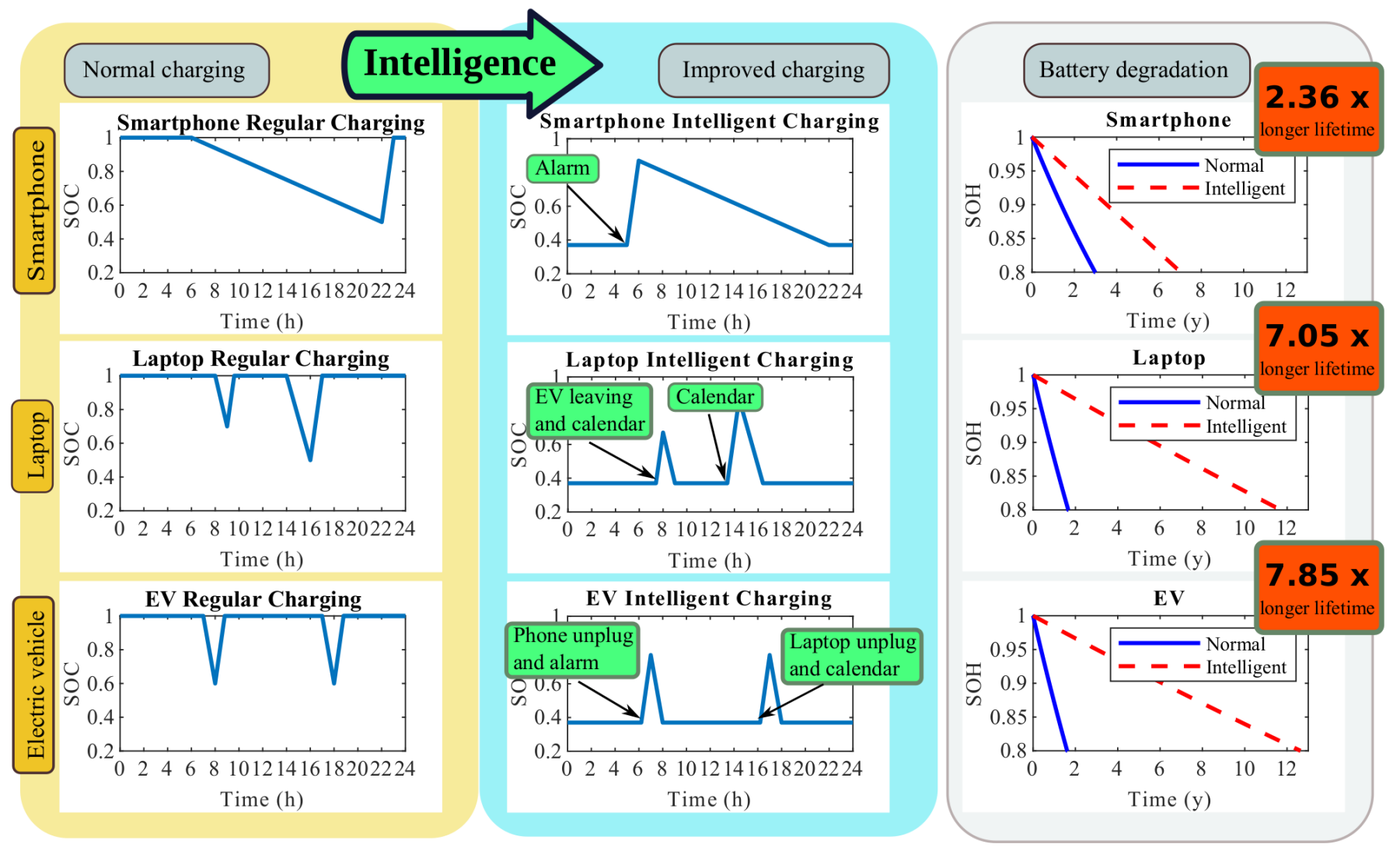

Fig. 4. Significant gains in battery SOH, i.e., the percentage of remaining battery capacity, can be achieved if intelligent charging with inter-device information exchange is applied.

\section{Standardized interface}

A wide range of different charger chips with different interfaces used in various phones further complicates the scenario. To address this, [2] proposes a remote-controlled switch (Figure 3(b)) to delay charging. Remotely controlled switches and power outlets are possible implementations with the advantage of being independent of the phone type. Although implementing intelligent chargers in principle will be possible without the help of manufacturers, the required additional hardware will make the resulting solutions cumbersome and less user friendly. If we want to optimize the charging of multiple devices, standardization across device classes would significantly simplify software reuse across device classes and should, therefore, be encouraged.

\section{THE ROAD AHEAD AND MAIN LESSONS}

Having discussed the high-level idea of intelligent chargers and having highlighted some of the challenges involved in building one, in the following, we summarize the main issues on the road ahead and key lessons learned in the following.

Since commercial solutions are not yet mature in this domain, there is considerable potential for innovation. Towards this, we made the following key findings. (i) Our case study reveals that there is a big potential to improve aging. (ii) Prediction is important to achieve those significant cycle life gains. Information such as usage context and location needs to be extracted to predict in particular the unplug time and target SOC. (iii) Inter-device usage information can help to further increase the accuracy of predictions and to mitigate the aging of multiple device classes. (iv) Individualized solutions can be developed thereby maximizing the cycle life gain. (v) HESSs can help to achieve additional gains in cycle life if an appropriate charge management strategy is applied.

Several challenges remain before such intelligent chargers will be broadly available. In this perspective paper, we have highlighted the next steps required. (i) We need to first identify and then extract information for high-quality prediction results at minimal computation and energy cost. (ii) Most importantly, we always need to maintain user comfort and user acceptance in any charger and predictor design. (iii) Further improved charge protocols that include online updates based on observed SOH development will, even more, increase cycle life. (iv) Finally, we need to simplify inter-device charging by standardization.

\section{ACKNOWLEDGMENT}

This work was supported by the National Research Foundation of Korea (NRF) grant funded by the Korea government (MSIP) (No. NRF- 2018R1A2B3007894). 
Alma Pröbstl received the Diploma in electrical and computer engineering from the Technical University of Munich, Munich, Germany, in 2013, where she is currently a Research Associate working towards her Ph.D. degree. Her current research interests include battery health management systems, energy storage systems, electromobility and power management.

Bashima Islam received the B.Sc. degree in computer science and engineering from Bangladesh University of Engineering and Technology, Dhaka, Bangladesh. She is currently pursuing the Ph.D. degree at the University of North Carolina at Chapel Hill, Chapel Hill, NC, USA. Her research interests include low-power computing and machine learning in resource-constrained devices.

Shahriar Nirjon is an assistant professor in the Department of Computer Science at the University of North Carolina at Chapel Hill, NC, USA. $\mathrm{He}$ is interested in building intelligent cyber-physical systems that involve embedded sensors and mobile devices, mobility and connectivity, and mobile data analytics.

Naehyuck Chang is currently a Full Professor with the School of Electrical Engineering, Korea Advanced Institute of Science and Technology. His current research interests include low-power embedded systems and the design automation of things. He is an ACM Fellow and an IEEE Fellow for the contribution to low-power design.

Samarjit Chakraporty (Senior Member, IEEE) is currently a William R. Kenan, Jr. Distinguished Professor in the Department of Computer Science at the University of North Carolina at Chapel Hill, NC, USA. His research interests include distributed embedded systems, embedded control systems, energy storage systems, electromobility, and sensor network-based information processing.

\section{REFERENCES}

[1] S. Saxena, G. Sanchez, and M. Pecht, "Batteries in portable electronic devices: A user's perspective," IEEE Industrial Electronics Magazine, vol. 11, no. 2, pp. 35-44, 2017.

[2] A. Pröbstl, P. Kindt, E. Regnath, and S. Chakraborty, "Smart2: Smart charging for smart phones," in Proceedings of RTCSA, 2015.

[3] J. Kim, A. Pröbstl, S. Chakraborty, and N. Chang, "Aging mitigation of power supply-connected batteries," in Proceedings of ISLPED, 2014.

[4] A. Bocca, A. Sassone, A. Macii, E. Macii, and M. Poncino, "An agingaware battery charge scheme for mobile devices exploiting plug-in time patterns," in Proceedings of ICCD, 2015.

[5] Y. Chen, A. Bocca, A. Macii, E. Macii, and M. Poncino, "A Li-ion battery charge protocol with optimal aging-quality of service trade-off," in Proceedings of ISLPED, 2016.

[6] L. He, Y.-C. Tung, and K. G. Shin, "iCharge: User-interactive charging of mobile devices," in Proceedings of MobiSys, 2017.

[7] A. Hoke, A. Brissette, K. Smith, A. Pratt, and D. Maksimovic, "Accounting for Lithium-ion battery degradation in electric vehicle charging optimization," IEEE Journal of Emerging and Selected Topics in Power Electronics, vol. 2, no. 3, pp. 691-700, 2014.

[8] K. Vatanparvar and M. A. Al Faruque, "Battery lifetime-aware automotive climate control for electric vehicles," in Proceedings of DAC, 2015.

[9] Q. Xie, X. Lin, Y. Wang, M. Pedram, D. Shin, and N. Chang, "State of health aware charge management in hybrid electrical energy storage systems," in Proceedings of DATE, 2012.

[10] H. Falaki, R. Mahajan, and D. Estrin, "Systemsens: a tool for monitoring usage in smartphone research deployments," in Proceedings of MobiArch, 2011.

[11] D. Ferreira, A. K. Dey, and V. Kostakos, "Understanding humansmartphone concerns: a study of battery life," in International Conference on Pervasive Computing, Springer, 2011.

[12] V. Doan, V. Vu, H. Vu, D. Tran, and W. Choi, "Intelligent charger with online battery diagnosis function," in Proceedings ofICPE-ECCE Asia, pp. 1644-1649, 2015. 